\title{
Molecular-dynamics ensembles: Fluctuations and correlations near the phase transitions
}

\author{
Juan J. Morales and María J. Nuevo \\ Departamento de Física, Facultad de Ciencias, Universidad de Extremadura, 06071 Badajoz, Spain \\ Luis F. Rull \\ Departamento de Fisica Atomica Nuclear y Molecular, Facultad de Física, Universidad de Sevilla, 41080 Sevilla, Spain
}

(Received 10 August 1992; revised manuscript received 30 November 1992)

\begin{abstract}
Computer simulations of liquid and solid systems very close to the melting-freezing transition zone have been performed for the microcanonical, canonical, and isothermal-isobaric molecular-dynamics ensembles. Temperature, pressure, and density fluctuations were studied over long evolution times, and graphical and analytical statistical-error methods were used to investigate correlations in the data. The Nose-Hoover (NH) method combined with the Toxvaerd algorithm is proposed as a correct method of obtaining the true fluctuation and correlation of the thermodynamic variables in the system, because the temperature and/or pressure constraints in the $\mathrm{NH}$ method do not affect the dynamical evolution of the system, and because the fifth-order Toxvaerd algorithm gives very accurate behavior for the correlations, as has been shown in recent studies.
\end{abstract}

\section{INTRODUCTION}

Interest in the statistical errors of computer experiments has increased over the past few years. The first problem was the theoretical estimate of the inherent error when sampling averages are replaced by averages over a finite time interval in evaluating the correlation function of stochastic (Gaussian) processes. ${ }^{1}$ The results of this study were confirmed in the case of the velocity autocorrelation function, ${ }^{2}$ and further application was made to the number density and kinetic-energy density autocorrelation functions for liquid sodium, ${ }^{3}$ and to a more general analysis that does not employ the Gaussian assumption. ${ }^{4}$ The topic has recently been taken up again and the predictions of the earlier work ${ }^{1}$ have been compared with simulation data of saturated liquid Ar in equilibrium. ${ }^{5}$ The initial problem of the inherent error was overcome with the generally accepted assumption of ergodicity, when the sampling average is equal to the time average in the limit of long times of calculation. Then computer simulation by either Monte Carlo (MC) (sampling-average) or by molecular-dynamics (MD) (time-average) methods are equally capable of giving the true thermodynamic behavior of the physical system with a good estimate of the statistical errors.

The problem of the persistence of correlation in long MC chains must be taken into account in estimating errors. ${ }^{6}$ This can be done using a correlation length as a measure of the persistence. One also $\mathrm{saw}^{7}$ how the sampling procedure in a calculation can be modified to minimize the variance of the distribution of a given variable. A treatment of the correlation comparing the efficiency of the MC and MD methods showed ${ }^{8}$ how the first two moments of the autocorrelation function of a variable along the chain are related to the expected variance of its mean. In that study, the variance of the potential energy in the canonical ensemble $(T, V, N)$ was shown to be larger than that in the microcanonical ensemble $(E, V, N)$ by a factor which is the ratio of the system heat capacity to that of an ideal gas.

Fluctuations of the thermodynamic parameters are related to the correlations. A theoretical study has been made of the equilibrium values, and their fluctuations, of some variables (temperature, pressure, and chemical potential) in different ensembles and then applied to the special case of computer experiments for the $(E, V, N)$ and $(T, V, N)$ ensembles. The results showed that, because of the finiteness of the systems, there is no unique solution when generalizing thermodynamic formulas from macroscopic systems to small systems. Interpreting the pressure mechanically and not just thermodynamically made it possible to evaluate the solutions physically. The study did not consider zones of large fluctuations such as the phase transitions and critical points. ${ }^{9}$

Several papers, therefore, have worked towards giving a general procedure for estimating the true variance of the mean of partially correlated measurements having finite $^{10}$ as well as infinite range correlations ${ }^{11}$ either in $\mathrm{MD}^{12}$ or MC. ${ }^{13}$

Traditionally, MD has been restricted to the microcanonical ensemble $\operatorname{MD}(E, V, N)$, but in response to experimental necessities other ensembles have come to be important. An example is the canonical $\operatorname{MD}(T, V, N)$ since, when calculating small pressures, it is important to use constant $T$ because the small differences between the required and actual temperature, unavoidable with the conventional $\operatorname{MD}(E, V, N)$, modify the ideal-gas contribution causing substantial systematic errors in the final results. $^{14}$

Thus, the different behavior of correlation functions and fluctuations for different $\mathrm{MD}$ ensembles is a significant problem for practical applications. In principle, the relation of fluctuation and correlations to extensive and intensive thermodynamic quantities can be evaluated analytically. ${ }^{15}$ The problem becomes severe if a system shows strong fluctuations, causing dominant finite 
size effects. ${ }^{16}$ The choice of the $2 \mathrm{D}$ melting for study is interesting because, among other things, the nature of the transition is still disputed and the accuracy of the method is crucial for this topic.

The main concern of the present paper is to investigate near the melting zone the temperature, pressure, and density fluctuations and correlations in detail for a twodimensional system using the recent isothermal-isochoric and isothermal-isobaric MD ensembles. A short account summarizing some of the important aspects of this work has appeared in Ref. 17. In Sec. II, we describe briefly the two statistical error theories used in our simulations. In Sec. III, we set up the starting conditions and the characteristics of the systems when using the NoséHoover ${ }^{18,19}$ method $(\mathrm{NH})$ to simulate the $\operatorname{MD}(E, V, N)$, $\operatorname{MD}(T, V, N)$, and $\operatorname{MD}(T, p, N)$ ensembles. This leads us to the study of the fluctuations and the correlations in Sec. IV. The discussion of the method, conclusions and final remarks are given in Sec. V.

\section{THE STATISTICAL ERROR METHODS}

A system is in equilibrium with respect to a variable $x$ when the mean and the variance of $x(t)$ are independent of time. The uncertainty in the mean is due to the fluctuation $\delta x(t)$. These fluctuations are a real property of the system, and the time average of $(\delta x)^{2}$ is also a thermodynamic quantity in which the ensemble corrections for mean fluctuations are significant.

Let $x_{i}=1, \ldots, n$ be the result of $n$ consecutive equally spaced measurements of some fluctuating quantity in a MD simulation of a system in equilibrium. The only assumption we make is that the correlations have a finite range, with no other a priori knowledge of their nature. The usual procedure is to obtain a numerical estimate by taking the mean $\bar{x}$, the variance $\sigma^{2}(x)$, and the variance of the mean $\sigma^{2}(\bar{x})$, but there are two possibilities for the statistical treatment: either considering a raw-data model or a block-data model. In the first method one has ${ }^{20}$

$$
\begin{aligned}
& \bar{x}=\frac{1}{n} \sum_{i=1}^{n} x_{i} \\
& \sigma^{2}\left(x_{i}\right)=\operatorname{var}\left(x_{i}\right)=\frac{1}{n} \sum_{i=1}^{n}\left(x_{i}-\bar{x}\right)^{2}, \\
& \sigma^{2}(\bar{x})=\operatorname{var}(\bar{x})=\frac{\sigma^{2}\left(x_{i}\right)}{n}(1+2 \tau),
\end{aligned}
$$

where $\tau$, termed the correlation length, is defined as

$$
\tau=\sum_{k=1}^{n-1} \hat{r}_{k}=\sum_{k=1}^{n-1} \frac{n-k}{n} \frac{\operatorname{cov}\left(x_{i}, x_{i-k}\right)}{\operatorname{var}\left(x_{i}\right)},
$$

$\hat{r}_{k}$ being the autocorrelation function at lag $k$. The variance and covariance are denoted by var and cov. Thus $\tau$ is the coefficient which determines the strength of correlation, with the value zero for uncorrelated values. If correlation exists, the effective number of independent values should be $n /(1+2 \tau){ }^{7}$ The second possibility consists in dividing the series of points $x_{i}$ into sequential nonoverlapping segments $n_{b}$. Each segment has $m$ points, and the length of each segment is $m \Delta t$, where $\Delta t$ is the time interval for obtaining the computer data. The value of $m$ will be chosen according to statistical considerations, as follows. The value of the mean for each block, the variance of the means, and the overall mean are given, respectively, by

$$
\begin{aligned}
& \bar{x}_{b}=\frac{1}{m} \sum_{i=1}^{m} x_{i}, \\
& \sigma^{2}\left(\bar{x}_{b}\right)=\operatorname{var}\left(\bar{x}_{b}\right)=\frac{1}{n_{b}} \sum_{b=1}^{n_{b}}\left(\bar{x}_{b}-\bar{x}\right)^{2}, \\
& \bar{x}=\frac{1}{n_{b}} \sum_{b=1}^{n_{b}} \bar{x}_{b}=\frac{1}{n} \sum_{i=1}^{n} x_{i},
\end{aligned}
$$

where $\bar{x}$ has the same value as in Eq. (1). Now Eq. (3) can be applied to the blocks as in the first method ${ }^{12}$ (note that the raw-data method is a particular case of the block-data method when $m=1$ and $n_{b}=n$, since $n=n_{b} m$ ). But our interest was not in obtaining another analytical method, but rather a graphical method, for which we used the Friedberg and Cameron idea $^{21}$ of plotting $m \operatorname{var}\left(\bar{x}_{b}\right) / \operatorname{var}\left(x_{i}\right)$ against $m$ until a plateau is reached. This plateau value is called the statistical inefficiency $I_{S}$ and fixes the appropriate block length $m .^{22}$

The relationship between the statistical inefficiency and the correlation length was found to $\mathrm{be}^{23}$

$$
I_{S}=1+2 \tau
$$

giving an equivalence between the graphical and the analytical methods.

\section{THE ENSEMBLES AND THE SYSTEMS}

We have performed long-run MD simulations near the melting-freezing transition for the $\operatorname{MD}(E, V, N)$, $\operatorname{MD}(T, V, N)$, and $\operatorname{MD}(T, p, N)$ ensembles in order to study the fluctuations and correlations for the thermodynamic variables of interest. Among the several procedures to simulate these ensembles we have chosen the NH method because it has been previously found ${ }^{24}$ that the constraints have no influence on the time behavior of the system. In this method, the particles are coupled to the corresponding reservoir by introducing isothermal $\zeta$ and isobaric $\dot{\varepsilon}$ thermodynamic friction coefficients which couple the system to the reservoir at $T=T_{\mathrm{ex}}$ and $p=p_{\mathrm{ex}}$, respectively. These coefficients are related to the corresponding relaxation times of the system $\tau_{T}$ and $\tau_{p}$. Both relaxation times have a Gaussian distribution and they are related to the mean collision time. ${ }^{24,25}$ The NH equations for a $D$-dimensional system are described by ${ }^{26}$

$$
\mathbf{x}_{i}^{\prime}=\mathbf{p}_{i} / m V^{1 / D}
$$

and

$$
\mathbf{p}_{i}^{\prime}=\mathbf{f}_{i}-\left(\varepsilon^{\prime}-\zeta\right) \mathbf{p}_{i},
$$

where $\mathbf{x}_{i}=\mathbf{r}_{i} / V^{1 / D}$ are the reduced coordinates for the particle $i, \mathbf{f}_{i}$ are the forces, and $\varepsilon^{\prime}=V^{\prime} / D V$. The time derivative of Eq. (10) is 
TABLE I. Systems simulated in solid $(S 1-S 3)$ and liquid $(L 1-L 3)$ using the microcanonical $(E, V, N)$, canonical $(T, V, N)$ and isothermal-isobaric $(T, p, N)$ ensembles. The final results for temperature, pressure, and density, after $N_{t}$ time steps, are shown with their standard deviations.

\begin{tabular}{lcccccr}
\hline \hline Ensemble & System & $N$ & $k T / \varepsilon$ & $p \sigma^{2} / \varepsilon$ & $\rho \sigma^{2}$ & $10^{3} N_{t}$ \\
\hline$(E, V, N)$ & $S 1$ & 256 & $1.07 \pm 0.02$ & $6.71 \pm 0.17$ & 0.9048 & 80 \\
$(T, V, N)$ & $S 2$ & 256 & $1.0001 \pm 0.0015$ & $6.09 \pm 0.05$ & 0.9048 & 90 \\
$(T, p, N)$ & $S 3$ & 256 & $1.0000 \pm 0.0011$ & $6.053 \pm 0.003$ & $0.9008 \pm 0.0009$ & 80 \\
$(E, V, N)$ & $L 1$ & 256 & $0.677 \pm 0.010$ & $2.46 \pm 0.08$ & 0.8260 & 114 \\
$(T, V, N)$ & $L 2$ & 400 & $0.7000 \pm 0.0008$ & $2.60 \pm 0.12$ & 0.8260 & 118 \\
$(T, p, N)$ & $L 3$ & 400 & $0.7000 \pm 0.0007$ & $2.641 \pm 0.002$ & $0.827 \pm 0.008$ & 118 \\
\hline \hline
\end{tabular}

$$
\mathbf{p}_{i}^{\prime \prime}=\mathbf{f}_{i}^{\prime}-\left(\varepsilon^{\prime \prime}-\zeta^{\prime}\right) \mathbf{p}_{i}-\left(\varepsilon^{\prime}-\zeta\right) \mathbf{p}_{i}^{\prime}
$$

where

$$
\zeta^{\prime}=\left(\sum_{i=1}^{N} \frac{\mathbf{p}_{i}^{2}}{m}-N D k T\right) / N D k T \tau_{T}
$$

and

$$
\varepsilon^{\prime \prime}=\left(p-p_{\mathrm{ex}}\right) V /\left(k T \tau_{p}^{2}\right) .
$$

To integrate the equation of motion, a very accurate fifth-order predictor-corrector algorithm, due to Toxvaerd, ${ }^{27}$ was used. This algorithm calculates the position and velocities of the particles up to fifth and third order in $h$ (the MD time step), respectively. That is,

$$
\begin{aligned}
\mathbf{r}_{i}(t+h) & =2 \mathbf{r}_{i}(t)-\mathbf{r}_{i}(t-h)+h^{2} \mathbf{a}_{i}(t) \\
& +\frac{1}{12} h^{4} \mathbf{a}_{i}^{\prime \prime}(t)+O\left(h^{6}\right)
\end{aligned}
$$

and

$$
\mathbf{r}_{i}^{\prime}(t)=\left[\mathbf{r}_{i}(t+h)-\mathbf{r}_{i}(t-h)\right] / 2-\frac{1}{6} h^{2} \mathbf{a}_{i}^{\prime}(t)+O\left(h^{4}\right) .
$$

In our simulation the time step was $h=0.005\left(m \sigma^{2} / \varepsilon\right)^{1 / 2}$, where $m, \sigma$, and $\varepsilon$ are the units of mass, length, and energy for the Lennard-Jones (12-6) potential. The Verlet ${ }^{28}$ criterion for the neighbors table was applied, with spherical and cutoff distances of $r_{\text {sd }}=2.75 \sigma$ and $r_{\text {co }}=2.5 \sigma$, respectively. The table was updated every $10 \mathrm{~h}$ and the usual periodic boundary conditions were used.

Two-dimensional systems were studied at two points of state: one defined by $k T / \varepsilon=1.0$ and $\rho \sigma^{2}=0.9048$, which correspond to the solid very close to the melting zone, ${ }^{29}$ and the other at $k T / \varepsilon=0.7$ and $\rho \sigma^{2}=0.8260$, which is a liquid very close to the freezing zone. ${ }^{30} \mathrm{We}$ have chosen these points because in previous simulations we found that the density fluctuations in the liquid ${ }^{26}$ for the $\operatorname{MD}(T, p, N)$ ensembles behave physically in the same way as the $T$ and $p$ fluctuations in the solid ${ }^{31}$ for the $\operatorname{MD}(E, V, N)$ ensemble, twice leading to a change of state and back. We now extend the study of the fluctuations to the correlations and to the three ensembles. Choosing conveniently the parameters in the $\mathrm{NH}$ equations one can reproduce the desired ensemble. Thus, the $\operatorname{MD}(E, V, N)$ ensemble was run with null isothermal $\zeta$ and isobaric $\varepsilon^{\prime}$ friction coefficients (isolated system), the $\operatorname{MD}(T, V, N)$ with $\varepsilon^{\prime}=0$ and isothermal relation time $\tau_{T}=0.005$ (system in contact with a thermal bath), and the $\operatorname{MD}(T, p, N)$ was $\tau_{T}=0.005$ and isobaric relaxation time $\tau_{p}^{*}=0.3$ (Ref. 26) (the system was in contact with a reservoir interchanging $T$ and $p$ ).

\section{THE FLUCTUATIONS AND CORRELATIONS}

Table I shows the mean values for $T, p$, and $\rho$, and the standard deviations for the various systems $(S 1, \ldots, L 3)$. The number of particles is $N$ and the total number of time steps $N_{t}$, the subaverages being taken every $800 \mathrm{~h}$. The relative standard deviations for $T$ and $p$ are very small when the NH method is used to fix these parameters. Moreover, these values are not appreciably different in solid and liquid, being about $0.1-0.15 \%$ for $T$ and $0.06-0.08 \%$ for $p$. For the systems where $T$ and $p$ are allowed to fluctuate, the standard deviations are significantly different in solid and liquid and depend on the ensemble as well. Thus the pressure uncertainties in $S 1$ and $S 2$ are about $2.5 \%$ and $0.82 \%$, respectively, which are about 45 and 15 times greater than in $S 3$, while for the liquid these values have risen to $3.3 \%$ and $4.6 \%$ for $L 1$ and $L 2$, which are about 45 and 60 times greater than for $L 3$.

In contrast with these results is the behavior of $T$ and $p$ in the $\operatorname{MD}(E, V, N)$ ensemble. Here the standard deviation for both properties is slightly smaller in the liquid than in the solid, thus not allowing the system to go back and forth between two points of state as in $S 1,{ }^{31}$ but permitting the oscillation of the fluctuations as can be seen in Fig. 1, where one can clearly see how the oscillations of the fluctuations in $T$ and $p$ are inverted as is expected in an isolated system such as the microcanonical ensemble.

As the density fluctuation for $L 3$ (Ref. 26) was found to behave in the same way as $T$ or $p$ in $S 1,{ }^{31}$ one can expect the same oscillating fluctuation for the density if the standard deviation decreases from liquid to solid in the same ratio as before. But the decrease is found to be about 10 times larger, and does not permit oscillations. The fluctuations for the other variables and ensembles are not significant, being a fairly regular sequence of peaks and valleys.

Because the state points chosen are very close to the phase transition where the fluctuations are very large, the study of correlations must be performed with great care in order to avoid inaccurate results. This was the reason for choosing the analytical ${ }^{12}$ and graphical ${ }^{21}$ methods described in Sec. II which, while very different in the way 


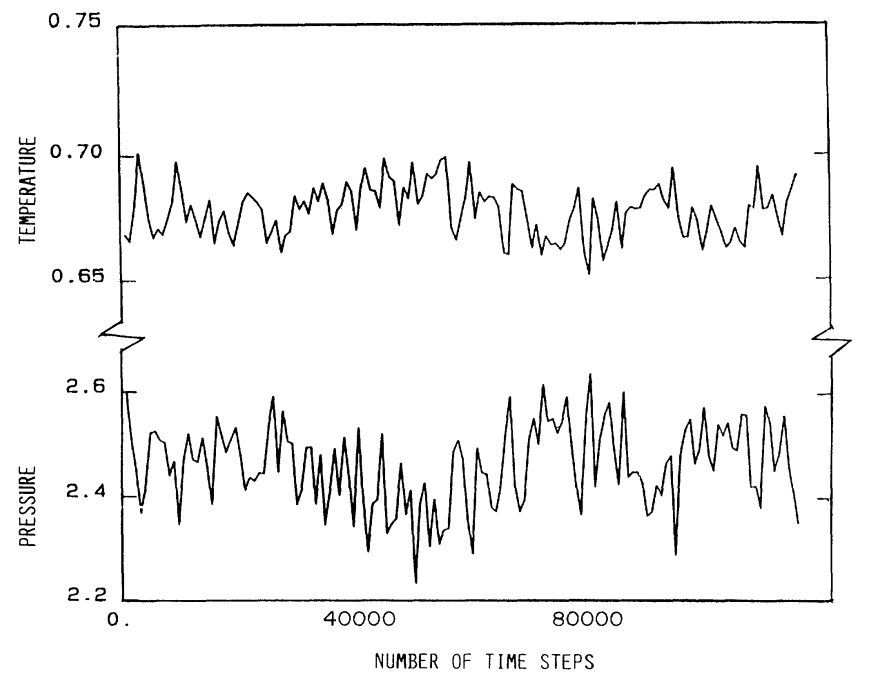

FIG. 1. Temperature (top) and pressure (bottom) fluctuations vs time steps, with the $\operatorname{MD}(E, V, N)$ ensemble in the liquid system $L 1$.

they obtain the correlations, must give the same results according to Eq. (8).

The first two systems studied were $S 1$ and $L 3$ because, although the variables which are permitted to fluctuate are different, the resulting fluctuations are similar. In Fig. 2, the temperature autocorrelation function $\hat{r}_{k}$ is plotted versus lag $k$ in $S 1$. For $\Delta t=800 \mathrm{~h}$, there is a steady fall from $k=1$ to 13 , but then there is a broad maximum at $k=15$ followed by a fall past zero at $k=23$. This behavior is also found for the other cases with the same height of the maxima, but occurring at smaller values of $k$ (about 7, 6, and 4, respectively) as a consequence of the sharper fall of the autocorrelation function. Applying the graphical method to obtain the statistical inefficiency $I_{S}$, we get Fig. 3, where one can see a pseudoplateau at about $m=12-17\left(I_{S}=8\right)$ before reaching the final plateau at $m=25$, giving $I_{S}=16$.

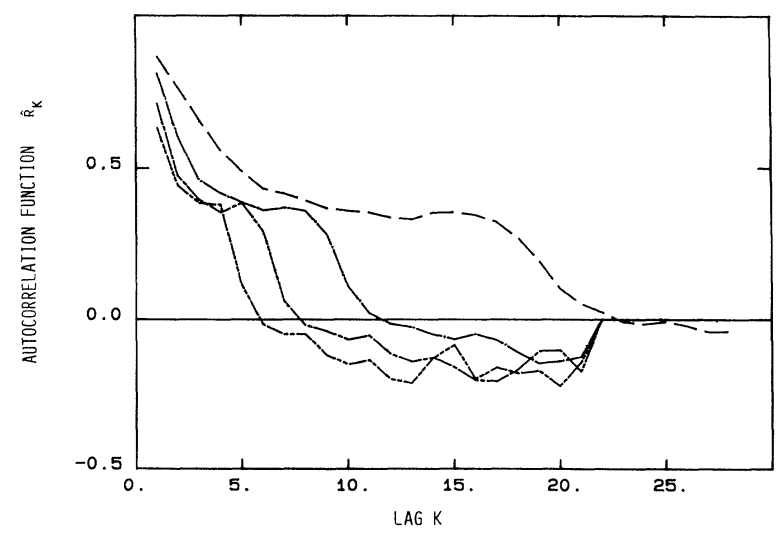

FIG. 2. Autocorrelation function $\widehat{r}_{k}$ vs lag $k$ for temperature, with the $\operatorname{MD}(E, V, N)$ ensemble in the solid system $S 1$. From top to bottom, the curves correspond to subaverages every $\Delta t=800,1600,2400$, and $3200 \mathrm{~h}$.

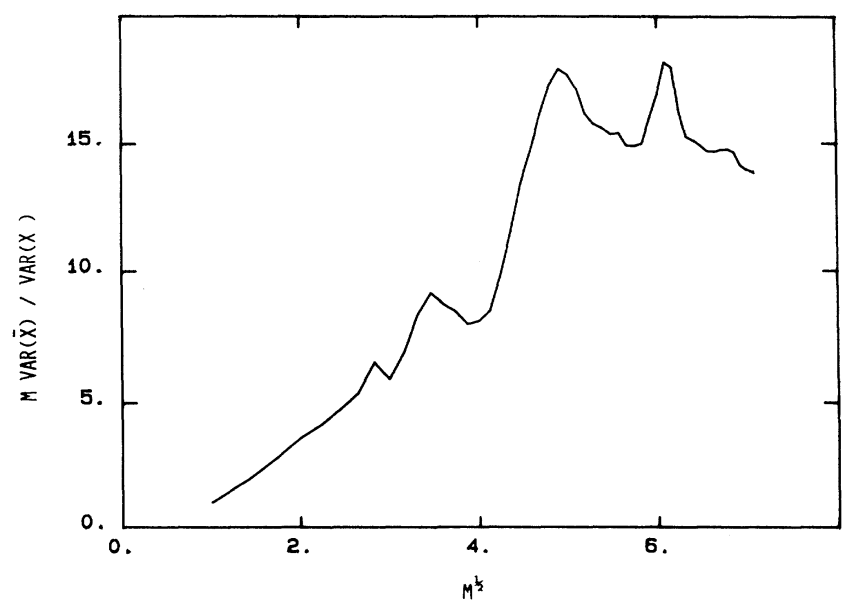

FIG. 3. Statistical inefficiency for temperature, with the $\operatorname{MD}(E, V, N)$ ensemble in the solid system $S 1$.

The maximum in the process of decay of the autocorrelation function (Fig. 2) and the pseudoplateau for the $I_{S}$ (Fig. 3) mean that stronger correlations appear in the system, which, however, disappear after a short time. The explanation of this behavior could be found, in principle, in the spontaneous fluctuations which drive the system between melting and solidifying as was shown in Ref. 31 . One should expect the same behavior for the density correlation in $L 3$, but as can be seen in Fig. 4 , the results are not the same. The fall for $\Delta t=800 \mathrm{~h}$ (upper line) is smoother and too long in comparison with the sharper decay and shorter period of oscillation when the number of time steps per subaverage is larger. When the graphical method is applied, the typical behavior of the $I_{S}$ (Fig. 5) appears, presenting only one plateau centered on $I_{S}=6$ for $m>9$. Thus the spontaneous fluctuations which appear in the liquid for the $\operatorname{MD}(T, p, N)$ ensemble are not seen in the correlation study with either of the two methods.

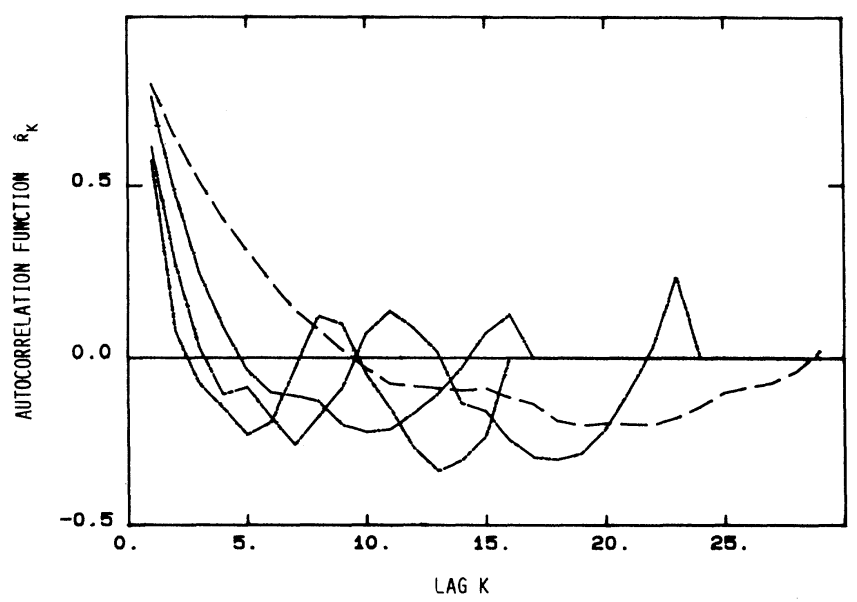

FIG. 4. Autocorrelation function $\widehat{r}_{k}$ vs lag $k$ for the density, with the $\operatorname{MD}(T, p, N)$ ensemble in the liquid system $L 3$. Curves as in Fig. 2. 


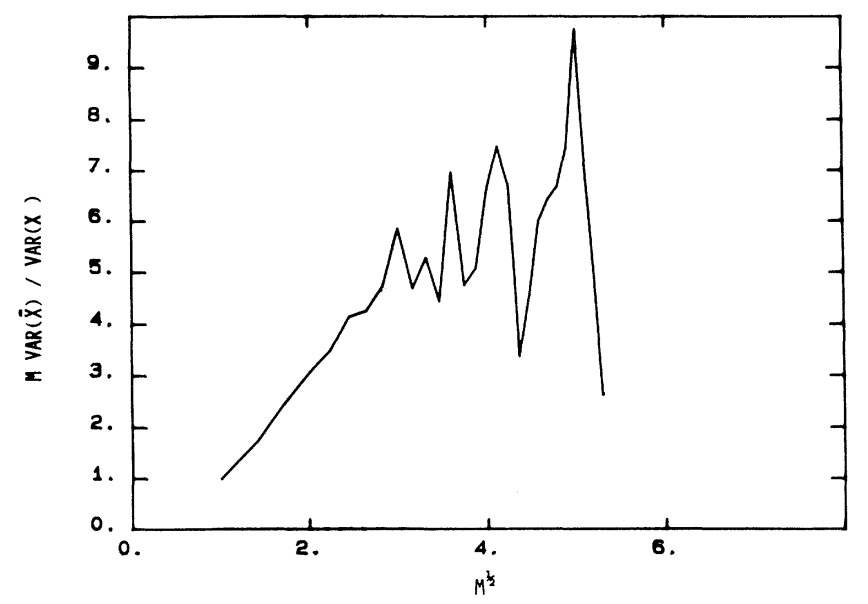

FIG. 5. Statistical inefficiency for density, with the $\operatorname{MD}(T, p, N)$ ensemble in the liquid system $L 3$.

For the remaining systems, only $L 1$ shows oscillation in the fluctuations for $T$ and $p$ (Fig. 1), the same oscillating behavior for the decay of $\widehat{r}_{k}$, and the plateau for the $I_{S}$. The other systems do not manifest any special behavior: $L 2$ has a sharper decay of the correlation and a smoother rise towards $I_{S}$, and there are no correlations for $S 2$ and $S 3$.

The values for $1+2 \tau$ and $I_{S}$ for the $T, p$, and $\rho$ for the various systems are collected in Table II. At first sight it seems that the $1+2 \tau$ values are always greater than the $I_{S}$ values when, in principle, the two methods should give the same values for every case, as in Eq. (8). The reason can be found in the intrinsic uncertainty of the methods. On the one hand, the graphical method introduces an uncertainty in $I_{S}$ because the plateau is reached after the onset of the fluctuations and the "noise"11 is progressively greater, as can be seen in Figs. 3 and 5. On the other hand, the problem of the analytical method is when to cut off the series of Eq. (4) which defines $\tau$. This is a widely studied problem, ${ }^{17,32,33}$ and we have used the common procedure which consists of including terms up to the order of $k$ at which $\hat{r}_{k}$ takes the first nearly zero value. The remaining terms of the series are thought to take oscillating values very close to zero with a null overall contribution to the correlation length. This criterion is a good approximation when there are no large

TABLE II. Statistical inefficiency $I_{S}$ and correlation length of the temperature, pressure, and density for the solid and liquid systems.

\begin{tabular}{cccc}
\hline \hline Variable & System & $1+2 \tau$ & $I_{S}$ \\
\hline$T$ & $S 1$ & 17.6 & 16.0 \\
$p$ & $S 1$ & 13.6 & 12.5 \\
$p$ & $S 2$ & 1.8 & 1.5 \\
$\rho$ & $S 3$ & 1.0 & 1.0 \\
$T$ & $L 1$ & 5.4 & 4.5 \\
$p$ & $L 1$ & 5.8 & 5.0 \\
$p$ & $L 2$ & 4.6 & 4.0 \\
$\rho$ & $L 3$ & 7.2 & 6.0 \\
\hline \hline
\end{tabular}

fluctuations of the thermodynamic variables under consideration, such as in the solid or liquid far from the phase transitions. Near these zones, however, the values of $\hat{r}_{k}$, once past the first zero, can be positive and negative not very close to zero, as can be seen in Figs. 2 and 4. It may be possible to calculate the amplitude of the noise, when a better approximation can be made, reducing the order in $k$ to that where $\hat{r}_{k}$ takes the first value close to that amplitude. This means that the series is cut off sooner than in the common procedure, lowering the value for $\tau$. Nevertheless, it is not always easy to find an oscillating behavior for the tail of $\hat{r}_{k}$ and one must either guess when the series must be cut off before the zero value is reached, or use the common procedure as before, but knowing in advance that the correlation length obtained will be somewhat greater than the real value.

\section{DISCUSSION AND CONCLUSIONS}

In a former study ${ }^{17}$ of the fluctuations and correlations in the $\operatorname{MD}(E, V, N)$ ensemble, we found a clear relationship between the two magnitudes: if the fluctuations around the mean values of the thermodynamic properties under study were too small or too large (with standard deviations about 0.001 and 0.1 , respectively) there were no correlations. In the former case, the system is not allowed to fluctuate because of the constraints on the ensembles, or because the point of state of the system is so stable that fluctuations are negligible. In the latter case, the amplitude of the fluctuations is so large that possible correlations between the data series are wiped out. For intermediate values of the standard deviation (about 0.01 ), there were correlations because the amplitudes of the fluctuations are at least smaller than the first value of the correlation series in Eq. (4). In the present cases the relationship is not so clear, and one conclusion from the results given in Sec. IV would be that every system must be studied separately. Thus, although the density fluctuations in the $\operatorname{MD}(T, p, N)$ for the $L 3$ system behave ${ }^{26}$ in the same was as the $T$ and $p$ fluctuations in the $\mathrm{MD}(E, V, N)$ for the $S 1$ system, ${ }^{31}$ the correlations do not behave the same: they are affected directly (Figs. 2 and 3) by the fluctuations in the $\operatorname{MD}(E, V, N)$ but are not affected (Figs. 4 and 5) in the $\operatorname{MD}(T, p, N)$. In the $\operatorname{MD}(T, V, N)$ ensemble, it is not possible to find any particular tendency in the system because the fluctuations in solid and liquid are large but regularly distributed in time with no special trend, so that the autocorrelation functions take values around zero from the first order in $k$.

The equivalence of the correlation length and statistical inefficiency methods is thus confirmed, but always while taking into account the kind of approximation used for the series cutoff in the analytical method and the inaccuracy introduced by the graphical method.

In general, with respect to the method used for the computer simulation of the $(T, V, N)$ and $(T, p, N)$ ensembles, in order to keep the total energy of the system constant, the velocities are usually rescaled every few time steps. Depending on the way of doing this, in some cases this form of temperature control does not keep the total momentum of the system zero, and for very long runs the 
random fluctuations eventually lead to an ever increasing total momentum for the system overall. The system is also weakly coupled to a heat bath both to keep $T$ near the present value and to keep the temperature from drifting upward due to numerical round-off error which always begins to accumulate whenever one makes runs longer than a few thousand time steps. ${ }^{34}$ Thus we need a simulation method which combines the positive aspects of these algorithms and avoids most of their negative aspects. That is, one needs an algorithm which is general enough that one can incorporate constant temperature and/or pressure methods while avoiding as far as possible energy drift during long simulations. The method we propose is the combination of the Nosé-Hoover method with the Toxvaerd algorithm. We have two strong arguments to defend this thesis. The first is that the $\mathrm{NH}$ method reproduces both the canonical and isothermalisobaric probability density in the phase space of an $\mathrm{N}$ body time-reversible classical mechanical system in equilibrium. ${ }^{35}$ This means that the NH equations obey a generalized Liouville equation and the general Langevin equation exactly, i.e., the constraints have no influence on the time behavior and one can perform long simulations with no problem, checking that the time-averaged values of the isothermal and isobaric friction coefficients are always zero. This statement has been tested by calculating the Mori coefficients ${ }^{24}$ and verifying that the friction coefficients have a Gaussian distribution. ${ }^{24,25}$ The second is that, since the fluctuations are mainly caused by the short-range interactions, one can diminish their effect by calculating very exactly the positions of the particles in the highly repulsive part of the potential, avoiding a significant drift in the energy. This can be performed by using the Toxvaerd algorithm. In our simulations the drifts were completely negligible $\left(\cong 10^{-7}\right)$. Thus, we have found that coupling the system to a heat bath, using the NH method and Toxvaerd algorithm, is a physically appealing way to reduce the effect of the numerical errors which accumulate during the course of any long simulation. The fluctuation obtained with this method gives the true fluctuation of the system. The correlations among the simulation data will depend on the accuracy of the algorithm used, as has recently been demonstrated ${ }^{36}$ by studying the influence of the MD algorithms on the calculated values of data correlations.

The importance of this kind of study has been confirmed with methods for implementing temperature and pressure controls at equilibrium and nonequilibrium to increase the efficiency of large-scale simulations in $\mathrm{NH}$ dynamics, ${ }^{37}$ the inclusion of an algorithm for the simulation of NH canonical molecular dynamics, ${ }^{38}$ the high precision shear-rate dependence of the viscosity of a Lennard-Jones liquid at the triple point using the $\mathrm{NH}$ method in nonequilibrium MD, ${ }^{39}$ and the application of these methods to Feynman's path integral theory. ${ }^{40}$

\section{ACKNOWLEDGMENT}

This research was partially supported by the CICYT, Project No. PB92-0523.
${ }^{1}$ R. Zwanzig and N. K. Ailawadi, Phys. Rev. 182, 280 (1969).

${ }^{2}$ D. Levesque and L. Verlet, Phys. Rev. A 2, 2514 (1970).

${ }^{3}$ N. K. Ailawadi, Phys. Rev. A 5, 1968 (1972).

${ }^{4}$ B. Picinbono, Phys. Rev. A 16, 2174 (1977).

${ }^{5}$ I. Bitsanis, M. Tirrell, and H. T. Davis, Phys. Rev. A 36, 958 (1987).

${ }^{6}$ W. W. Wood, in Physics of Simple Liquids, edited by H. N. V. Temperley, J. S. Rowlinson, and G. S. Rushbrook (NorthHolland, Amsterdam, 1968).

${ }^{7}$ L. D. Fosdick, Methods in Computational Physics, edited by B. Alder, S. Fernbach, and M. Rotenberg (Academic, New York, 1963), Vol. 1.

${ }^{8}$ G. Jacucci and A. Rahman, Nuovo Cimento D 4, 341 (1984).

${ }^{9}$ K. W. Kratky, Phys. Rev. A 31, 945 (1985).

${ }^{10}$ G. J. Daniell, A. J. G. Hey, and J. E. Mandula, Phys. Rev. D 30, 2230 (1984).

${ }^{11}$ H. Flyvbjerg and H. G. Petersen, J. Chem. Phys. 91, 461 (1989).

${ }^{12}$ S. K. Schifel and D. C. Wallace, J. Chem. Phys. 83, 5203 (1983).

${ }^{13}$ E. B. Smith and B. H. Wells, Mol. Phys. 53, 701 (1984).

${ }^{14}$ D. Fincham, N. Quirke, and D. J. Tildesley, J. Chem. Phys. 84, 4535 (1986).

${ }^{15}$ J. L. Lebowitz, J. K. Percus, and L. Verlet, Phys. Rev. 153, 250 (1967).

${ }^{16}$ M. S. S. Challa, D. P. Landau, and K. Binder, Phys. Rev. B 34, 1841 (1986).

${ }^{17}$ J. J. Morales, F. Cuadros, and L. F. Rull, J. Chem. Phys. 86, 2960 (1987)
${ }^{18}$ S. Nosé, Mol. Phys. 52, 255 (1984).

${ }^{19}$ W. Hoover, Phys. Rev. A 31, 1695 (1985).

${ }^{20}$ Monte Carlo Methods in Statistical Physics, 2nd ed., edited by K. Binder (Springer, Berlin, 1986).

${ }^{21}$ R. Friedberg and J. E. Cameron, J. Chem. Phys. 52, 6049 (1970).

${ }^{22}$ Another approach recommended in Computer Simulation of Liquids (Oxford University Press), by M. P. Allen and D. J. Tildesley, is to plot this quantity vs $m^{-1}$, when the intercept as $m^{-1}$ tends to zero gives the desired result.

${ }^{23}$ J. J. Morales, M. J. Nuevo, and L. F. Rull, J. Comput. Phys. 89, 432 (1990).

${ }^{24}$ L. F. Rull, J. J. Morales, and S. Toxvaerd, Phys. Rev. A 38, 4309 (1988).

${ }^{25}$ D. J. Evans and B. L. Holian, J. Chem. Phys. 83, 4069 (1985).

${ }^{26}$ J. J. Morales, M. J. Nuevo, and L. F. Rull, Phys. Rev. B 43, 3514 (1991).

${ }^{27}$ S. Toxvaerd, J. Comput. Phys. 47, 444 (1982).

${ }^{28}$ L. Verlet, Phys. Rev. 159, 98 (1967).

${ }^{29}$ S. Toxvaerd, Phys. Rev. A 24, 2735 (1981).

${ }^{30}$ S. Toxvaerd, Phys. Rev. B 29, 2821 (1984).

${ }^{31}$ L. F. Rull, J. J. Morales, and F. Cuadros, Phys. Rev. B 32, 6050 (1985).

${ }^{32}$ T. P. Straatsma, H. J. C. Berendsen, and A. J. Stam, Mol. Phys. 57, 89 (1986).

${ }^{33}$ S. Dietrich and H. Dette, J. Comput. Phys. 101, 224 (1992).

${ }^{34}$ K. Kremer and G. S. Grest, J. Chem. Phys. 92 (1990).

${ }^{35}$ S. Nosé, Prog. Theor. Phys. 103, 1 (1991).

${ }^{36}$ J. J. Morales, Phys. Lett. A 172, 215 (1993). 
${ }^{37}$ B. L. Holian, A. J. De Groot, W. G. Hoover, and C. G. Hoover, Phys. Rev. A 41, 4552 (1990).

${ }^{38}$ S. Toxvaerd, Mol. Phys. 72, 159 (1991).

${ }^{39}$ M. Ferrario, G. Ciccotti, B. L. Holian, and J. P. Ryckaert,
Phys. Rev. A 44, 6936 (1991).

40J. J. Morales and K. Singer, Mol. Phys. 73, 873 (1991); J. J. Morales and M. J. Nuevo (unpublished). 